\title{
LA IMPOSICIÓN SELECTIVA AL CONSUMO EN EL PERÚ: ENTRE EL FIN RECAUDATORIO Y LA EXTRAFISCALIDAD
}

\author{
THE SELECTIVE TAXATION OF CONSUMPTION IN PERU: BETWEEN \\ THE RECOVERY PURPOSE AND THE EXTRAFISCALITY
}

Raúl EnRiQue Ríos Culquicondori Universidad Nacional Agraria de la Selva Tingo María, Perú

ORCID: http://orcid.org/0000-0001-8217-2356

Correo electrónico: raen rc@hotmail.com

\section{RESUMEN}

Objetivo: El propósito de la investigación es determinar si los fundamentos de la imposición selectiva, su estructura, finalidad e importancia teórica se corroboran en la práctica. Para ello, se cuestiona si la modificación en mención fue con fines recaudatorios o si fue más bien, una medida desalentadora de consumo. Método: Es una investigación cualitativa ya que el fenómeno es explorado y descrito desde la perspectiva del investigador y se fundamenta en la revisión de literatura, en el contexto y en la intuición. Resultados: En mayo de 2018, el Ministerio de Economía y Finanzas dictó diversos decretos supremos incrementando las tasas del Impuesto Selectivo al Consumo en el Perú. Los productos afectados por el alza fueron los combustibles, las bebidas azucaradas, las bebidas alcohólicas, y los vehículos nuevos y usados. La disposición lejos de pasar desapercibida, se tornó controversial y mediática, poniendo en manifiesto la desinformación que existe sobre este tributo. Conclusiones: En la práctica la imposición selectiva se ve distorsionada en cuanto a los fundamentos teóricos que la sustentan, es decir, el impuesto no está cumpliendo su finalidad extrafiscal (desmotivar el consumo de los bienes afectos con el gravamen) sino en cambio, casi por inercia y apoyado en las características económicas de los bienes que son sujetos con el gravamen, el fin fiscal está superando a la extrafiscalidad tradicional que debe perseguir la imposición selectiva.

Palabras clave: Impuesto; selectivo; extrafiscalidad; recaudación; consumo.

\begin{abstract}
Objective: The purpose of the investigation is to determine whether the fundamentals of selective taxation, its structure, purpose and theoretical importance are corroborated in practice. To do this, it is questioned whether the modification mentioned was for collection purposes or if it was, rather, a discouraging measure of consumption. Method: It is a qualitative investigation since the phenomenon is explored and described from the perspective of the researcher and is based on the review of literature, in context and in intuition. Results: In May 2018, the Ministry of Economy and Finance issued several supreme decrees increasing the rates of the Selective Consumption Taxes in Perú. The products affected by the increase were fuels, sugary drinks, alcoholic beverages, and new and used vehicles. The disposition far from going unnoticed became controversial and media, revealing the misinformation that exists on this tribute. Conclusions: In practice, the selective taxation it shows distorted in terms of the theoretical fundaments that support it, that is to say, the tax is not accomplish its extra-fiscal purpose (demotivating the consumption of the goods affected by the burden) but instead, almost by inertia and supported by the economic characteristics of the goods that are subject to the burden, the fiscal purpose is to overcoming the traditional extrafiscality that must follow the selective taxation.
\end{abstract}

Keywords: Tax; selective; extrafiscalidad; collection; consumption.

(c) Los autores. Este artículo es publicado por la Revista Quipukamayoc, Universidad Nacional Mayor de San Marcos. Este es un artículo de acceso abierto, distribuido bajo los términos de la Licencia Creative Commons Atribución-NoComercial-Compartirlgual 4.0 Internacional.(http://creativecommons.org/licenses/by-nc-sa/4.0/), que permite el uso no comercial, distribución y reproducción en cualquier medio, siempre que la obra original sea debidamente citadas. 


\section{INTRODUCCIÓN}

El Estado moderno se basa en su poder de imperio -ius imperium- para ejercer, entre otras actuaciones, el poder tributario o la también llamada potestad tributaria, dicho poder se desarrolla en estricto cumplimiento del ordenamiento jurídico vigente y con el fin prioritario de obtener ingresos (fin fiscal) para cubrir los gastos que demanda la satisfacción de las necesidades públicas.

En síntesis, la potestad tributaria es la facultad que posee el Estado para exigir legalmente (a través de normas jurídicas) la transferencia de riqueza por parte de los particulares hacia o en favor de aquel. No obstante, Villegas (2001) refiere que:

El tributo puede perseguir también fines extrafiscales, o sea, ajenos a la obtención de ingresos (...). Este objetivo extrafiscal adicional (aun siendo la motivación prevaleciente de alguna medida tributaria) no constituye la esencia jurídica de la institución del tributo, sino la utilización para objetivos económico-sociales de una herramienta de intervencionismo. (p. 68)

Villegas (2001) agrega que, "el Estado puede intervenir activamente en la economía nacional orientándola en virtud de medidas tributarias, y es lógico que así lo haga, si de ello se generan beneficios a la comunidad organizada" (p. 68). Máxime si estos beneficios giran en torno a la protección del medio ambiente, la reducción del consumo de bebidas alcohólicas, cigarrillos y/o juegos de azar. En aras de prevenir efectos que potencialmente podrían derivar en trastornos sociales que alteren la armoniosa convivencia de los ciudadanos en la comunidad; y/o en enfermedades que generen una disminución en la calidad de vida de las personas.

\section{Características de la imposición selectiva al consumo}

El principal impuesto al consumo es el Impuesto General a las Ventas (IGV), el cual afecta de forma general la compra de bienes y adquisiciones de servicios que toda la población realiza a diario. No obstante, Ruiz de Castilla (2017) refiere que, "el IGV se encuentra acompañado de un impuesto a los consumos específicos, llamado Impuesto Selectivo al Consumo (ISC), que se aplica básicamente a la venta de combustibles, bebidas, cigarros y vehículos que realizan los fabricantes o importadores" (p. 74).

El ISC es un impuesto monofásico (a diferencia del IGV que se caracteriza por su plurifasidad), es decir, se grava una sola etapa de la cadena productiva; en el caso peruano, a nivel de productor o importador. Siendo estos últimos, los encargados de la declaración y pago del tributo, lo que en doctrina se conoce como sujeto pasivo del impuesto.
En este sentido, el impuesto se exige sobre la base del valor del producto, sobre una unidad de medida del mismo o sobre el valor según precio de venta al público. Estos sistemas se observarán a efectos de determinar la base imponible sobre la cual se aplicarán las tasas impositivas correspondientes.

Cabe resaltar, que el Tribunal Fiscal en su Resolución No 4076-4-2008 (como se cita en Alva, 2017), enfoca al ISC como un impuesto indirecto pues según precisa el colegiado: "el Impuesto Selectivo al Consumo es un impuesto indirecto pues incide económicamente sobre los consumidores en la medida que el contribuyente traslade el gravamen en el precio de venta de los bienes (...)". Si bien técnicamente el ISC en su configuración no contempla la traslación del impuesto a través de las diversas fases de producción y comercialización como ocurre en el caso del IGV, no obstante, dicha traslación no puede ser prohibida o imposibilitada, pasando a ser la misma, una decisión que depende solo y exclusivamente de los contribuyentes (productores o importadores) del impuesto.

\section{Finalidad del ISC}

Existe consenso en cuanto a la finalidad extrafiscal que persigue la imposición selectiva, es decir, la de buscar fundamentalmente desalentar el consumo de los bienes afectos mediante el incremento de sus precios en el mercado. Villanueva (2014) sostiene en relación al ISC que: “(...) la finalidad recaudatoria pasa a un segundo plano y que antes bien, el impuesto busca modificar la conducta de los consumidores" (p. 814). Aclara el citado autor que: “(...) en la imposición selectiva se busca incrementar el precio relativo de los productos a fin de desalentar en los consumidores el consumo de determinados bienes y servicios" (p. 814).

Sin perjuicio de lo anterior, algunos autores como Arias y Plazas (2002) advierten que existen varios fundamentos para la imposición selectiva al consumo, poniendo énfasis en las siguientes consideraciones:

a. Son una fuente importante de generación de ingresos (Objetivo Fiscal)

b. Permite la corrección de externalidades negativas (Objetivo Extrafiscal)

c. Son herramientas para mejorar la equidad vertical, es decir, afectar con más carga impositiva al que exterioriza mayor capacidad contributiva (Objetivo Fiscal)

Teniendo en cuenta lo expuesto, cuanto menos teóricamente es aceptada la noción que los gobiernos utilizan la imposición selectiva como parte de una política pública de intervencionismo en la economía, de allí que se 
le atribuya al ISC primordialmente un fin extrafiscal que busca guiar el comportamiento de los consumidores, al tratar de desincentivar el consumo de productos nocivos para la salud, lo cual fomenta el consumo de alternativas más saludables, la protección del medio ambiente, y además, constituye una fuente significativa de ingresos para el erario público.

\section{Bienes gravados con el ISC}

En atención a los fundamentos que sustentan la imposición selectiva, nace la interrogante del porqué de la elección de unos u otros bienes a efectos de ser objetos con el gravamen. Arias y Plazas (2002) sostienen que:

Los impuestos selectivos al consumo gravan bienes que presentan alguna de las siguientes características: a) bienes cuya producción y venta está supervisada de cerca por el gobierno, b) bienes con demanda inelástica, c) bienes con elasticidad ingreso mayor que uno (bienes suntuarios) y d) bienes cuyo consumo causan externalidades negativas (p. 9).

Pero las razones más importantes para la elección de unos bienes en relación de otros, radica en que "los bienes afectos (...), tienen grandes volúmenes de ventas, pocos productores, demanda inelástica, fácil diferenciación y ausencia de sustitutos cercanos" (Arias y Plazas, 2002, p. 10).

En consecuencia, Villanueva (2014), refiriéndose a los bienes sujetos al ISC, afirma que: "Estos productos se ven mínimamente afectados en su demanda ante el aumento de sus precios" (p. 825).

Como corolario, en nuestro sistema tributario existe una lista taxativa de bienes y/o servicios gravados, regulada en el artículo 50 de la Ley del IGV/ISC; que en resumen se subdividen en:

\section{$\checkmark$ Combustibles \\ $\checkmark$ Bebidas azucaradas \\ $\checkmark \quad$ Vehículos \\ $\checkmark$ Cigarros \\ $\checkmark \quad$ Juegos de Azar y apuestas \\ $\checkmark$ Bebidas alcohólicas}

El propósito de la investigación es determinar si los fundamentos de la imposición selectiva, su estructura, finalidad e importancia teórica se corroboran en la práctica. Para ello, se cuestiona si la modificación en mención fue con fines recaudatorios o si fue más bien, una medida desalentadora de consumo.

\section{MATERIAL Y MÉTODOS}

Es una investigación cualitativa, ya que el fenómeno es explorado y descrito desde la perspectiva del investigador y se fundamenta en la revisión de literatura, en el contexto y en la intuición. En este sentido, se construyó el marco teórico a través del cual se logró comprender los fundamentos, estructura e importancia de la imposición selectiva, con el fin determinar si la aplicabilidad práctica del tributo corresponde a sus fundamentos teóricos.

El artículo tiene un diseño de investigación no experimental, no obstante, las explicaciones expuestas se circunscriben a un ámbito determinado, poseen riqueza interpretativa $\mathrm{y}$ aportan nuevas visiones del fenómeno estudiado (Hernández y Mendoza, 2018). Las categorías de análisis que se determinó para el desarrollo de la investigación fueron la finalidad del ISC (objetivo extrafiscal y característica de los bienes gravados), la recaudación tributaria del ISC (recaudación tributaria antes y después de la modificación efectuada) y la variación de los precios de los bienes afectos con el ISC (antes y después de la modificación).

Los datos fueron recolectados en función a las categorías de análisis, utilizando como instrumento de recolección la revisión bibliográfica y el análisis documentario de la recaudación del ISC revelado anualmente por la Superintendencia Nacional de Aduanas y de Administración Tributaria(SUNAT) ylos precios promedio al consumidor en Lima Metropolitana presentados por el Instituto Nacional de Estadística e Informática (INEI). Los datos recolectados se organizaron y procesaron en Ms Excel y los mismos son sujetos a análisis interpretativos y comparativos que permitieron arribar a los resultados y las conclusiones de la investigación.

\section{RESULTADOS}

Bienes afectados con la modificación

El 9 de mayo de 2018, se publicó en el Diario Oficial El Peruano, cinco Decretos Supremos mediante los cuales se incrementaron las tasas del ISC de los bienes que se muestran en la tabla 1 .

La citada modificación entró en vigencia al día siguiente de su publicación de conformidad con lo señalado por el artículo $11^{\circ}$ de Ley Orgánica del Poder Ejecutivo: “(...) Los Decretos Supremos entran en vigencia al día siguiente 
Tabla 1

Detalle de la modificación efectuada al Impuesto Selectivo al Consumo.

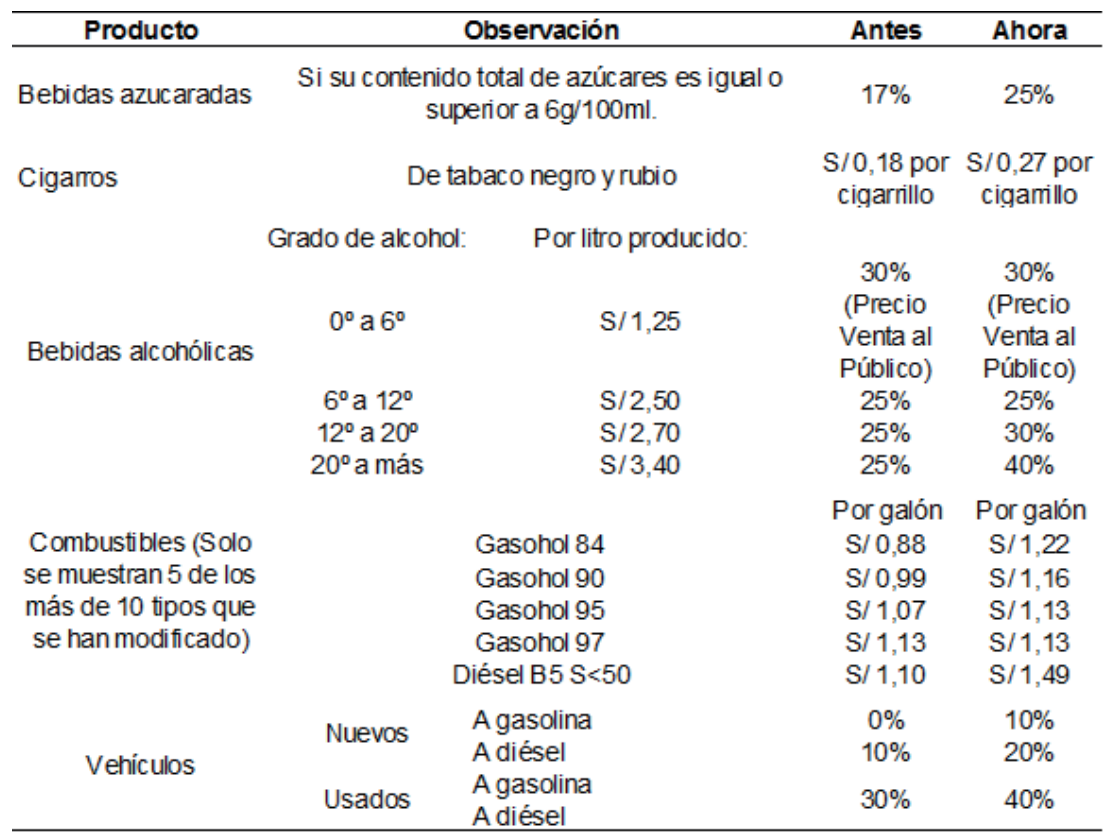

Fuente: Elaboración propia, en base a la información contenida en los Decretos Supremos No 091-2018-EF, No 092-2018-EF, No 093-2018-EF, No 094-2018-EF y No 095-2018-EF.

de su publicación en el Diario Oficial, salvo disposición contraria del mismo, que postergue su vigencia en todo o en parte". Dicha disposición no estuvo exenta de críticas, especialistas y un gran sector de la población, la tildaron como una medida políticamente inoportuna. ¿Aumento de la Remuneración Mínima Vital seguida del incremento en los precios de los bienes? ¿Medida abusiva ante la pasividad de la población? ¿Crecimiento económico a costa de alza en las tasas impositivas? ¿Más impuestos para seguir financiando actos de corrupción? Fueron algunas de las cuestiones que retumbaron en el inconsciente colectivo de la población, cuestiones válidas, desde la óptica del peruano cansado de la ineficacia de las autoridades y de las desigualdades económicas, pero tal vez no desde quién conoce realmente cuál es la función extrafiscal que cumple la imposición selectiva.

\section{Contexto Político-Fiscal}

Cuando se dictó la modificación materia de análisis, el país atravesaba una situación política sumamente crítica, el obstruccionismo legislativo, los conflictos de intereses y los destapes de escándalos de corrupción tuvieron como consecuencia cambios constantes en los integrantes de los distintos ministerios. En un periodo menor a los tres años se sustituyó al titular del Ministerio de Economía y Finanzas en seis oportunidades. El punto más álgido de este episodio fue la dimisión del expresidente Kuczynski y la consecuente asunción al cargo de Martin Vizcarra. Este contexto de fragilidad política, sumado a la disminución en la recaudación neta de tributos, propició que cada nuevo ministro encargado de la cartera de Economía y Finanzas llegara con un conjunto de medidas tributarias bajo el brazo, que se entiende, en aras de incrementar la recaudación en el más corto plazo posible.

Las medidas adoptadas en materia tributaria fueron de diversa índole: disminución de la tasa general del Impuesto a la Renta empresarial, la modificación de los tramos y tasas del Impuesto a la Renta a las rentas del trabajo y de fuente extranjera, la implementación del sistema de diferimiento de pago del IGV (denominado IGV justo); así como la creación del Régimen MYPE Tributario para empresas cuyos ingresos netos no superen las 1700 UIT.

Si bien estas medidas se dictaron con el objetivo de dinamizar la economía, ampliar la base tributaria y promover la formalidad, las mismas también contribuyeron a una reducción en la recaudación de impuestos. Así lo reconoce la propia SUNAT (2018) al manifestar que, " entre los factores que incidieron en la disminución de la recaudación, el efecto de algunas medidas tributarias como el diferimiento del Pago del IGV y el nuevo régimen del Impuesto a la Renta para MYPES” (p. 23). 


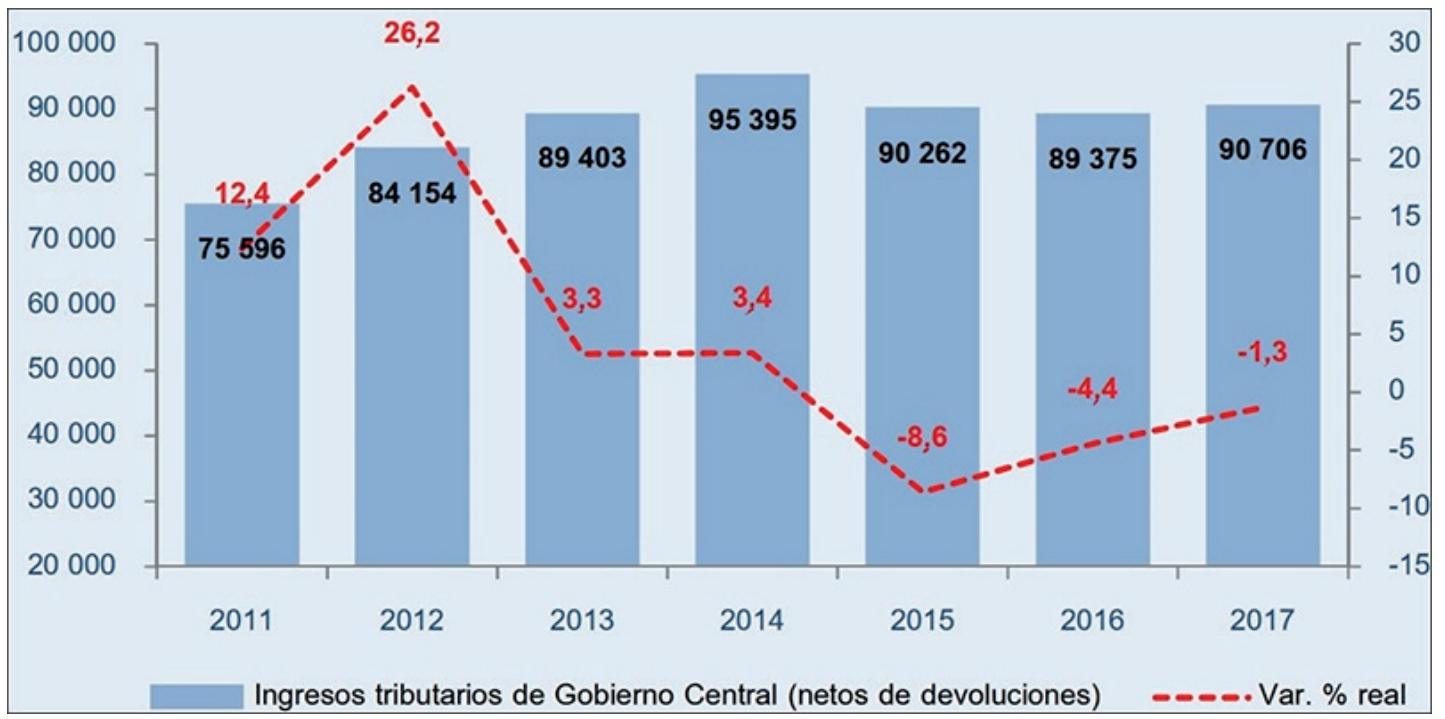

Figura 1. Ingresos tributarios del gobierno central (netos de devoluciones). En millones de soles y variación (\%). Fuente: SUNAT: División de Estadística - Gerencia de Estudios Económicos.

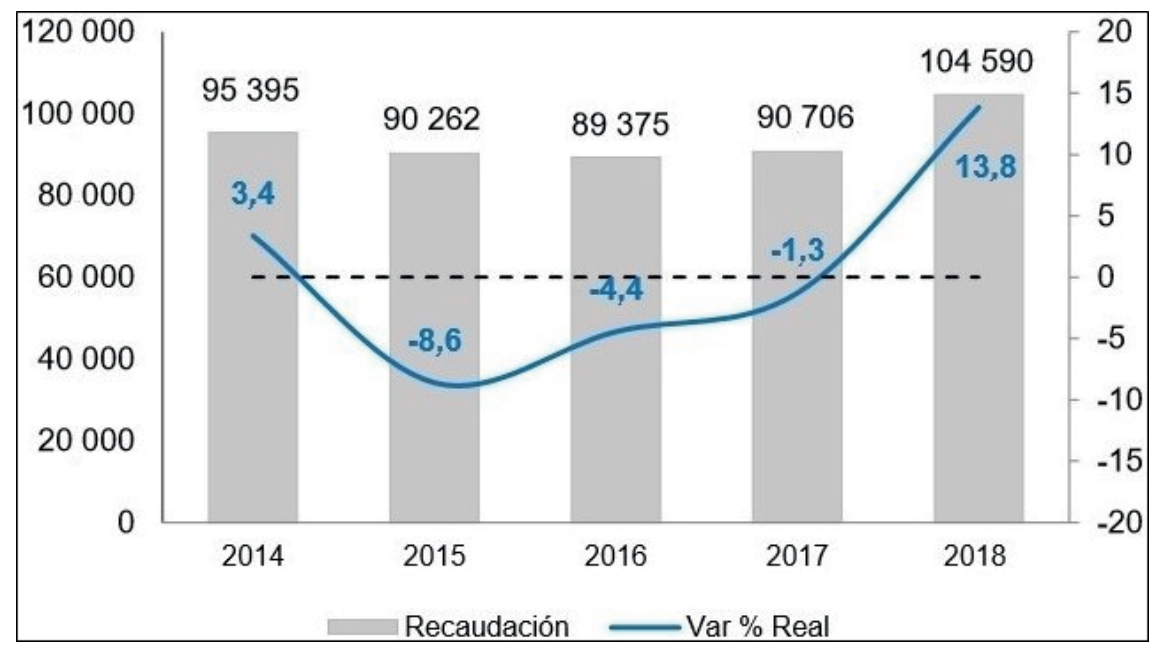

Figura 2. Ingresos tributarios Netos 2014 - 2018 (En millones de soles y Var. \% real anual). Fuente: SUNAT: División de Estadística - Gerencia de Estudios Económicos.

\section{Impacto en la recaudación luego de la modificación}

En contraposición a los resultados mostrados en la Figura 1, el año 2018 registró resultados muy notables en la recaudación: "En el año 2018 se recaudaron S/ 104590 millones de ingresos tributarios del Gobierno Central (descontando las devoluciones de impuestos); dicho importe representó S/ 13884 millones más que en 2017 (...)” (SUNAT, 2019, p. 22) (ver figura 2).

SUNAT (2019) afirma que: "En términos generales, los resultados obtenidos en el año 2018 fueron explicados por diversos factores entre los cuales se puede mencionar como principales (...) el incremento de las tasas impositivas del ISC aplicado desde mayo 2018” (p. 43).

Además, afirma que: "La recaudación por concepto del ISC sumó S/ 6860 millones; lo que significó un crecimiento de 7,2\% y una mayor recaudación de S/ 545 millones respecto del 2017" (p. 46).

SUNAT (2019) concluye que:

El comportamiento del ISC durante el año 2018 se debe no sólo a la evolución de la actividad económica 
(4,0\%) y demanda interna (más de 4\%), sino principalmente al incremento de tasas impositivas aplicadas a un grupo importante de productos desde mayo de 2018 (combustibles, licores, bebidas azucaradas, cigarros, vehículos, cervezas, entre otros) (p. 47).

Impacto en la variación de los precios

En la Tabla 2, se presenta un análisis no exhaustivo de la variación de los precios de tres productos (tomados como muestra) afectos con el ISC. Para ello, se tomó como punto de partida el año 2014 en el que se evidenció la caída más llamativa en la recaudación de este impuesto (Véase la Figura 3).

En la tabla 2, se observa una tendencia al alza en los precios de los productos gravados con el ISC, sin embargo, la Figura 3 muestra un aumento en la recaudación de dicho tributo en el mismo periodo analizado. Este comportamiento resulta contrario a los fundamentos que apoyan la imposición selectiva al consumo. Por lo tanto, el análisis comparativo permite cuanto menos a priori, afirmar que las modificaciones efectuadas tuvieron, principalmente, efectos recaudatorios antes que desmotivadores de consumo.

\section{DISCUSIÓN}

La teoría sostiene que, la imposición selectiva al consumo busca aumentar los precios de los bienes para desmotivar su consumo, es decir, disminuir la cantidad demandada. Empero, por principios económicos al disminuir la cantidad demandada los productores tienden a ajustar su producción (oferta) propiciando, en este sentido, que la recaudación disminuya o en el mejor de los casos, se mantenga constante. Sin embargo, el comportamiento antes descrito no se evidencia en los resultados obtenidos, por el contrario, el aumento del gravamen se encuentra acompañado por el aumento en la recaudación del impuesto estudiado. Esta situación, confirma lo ase-

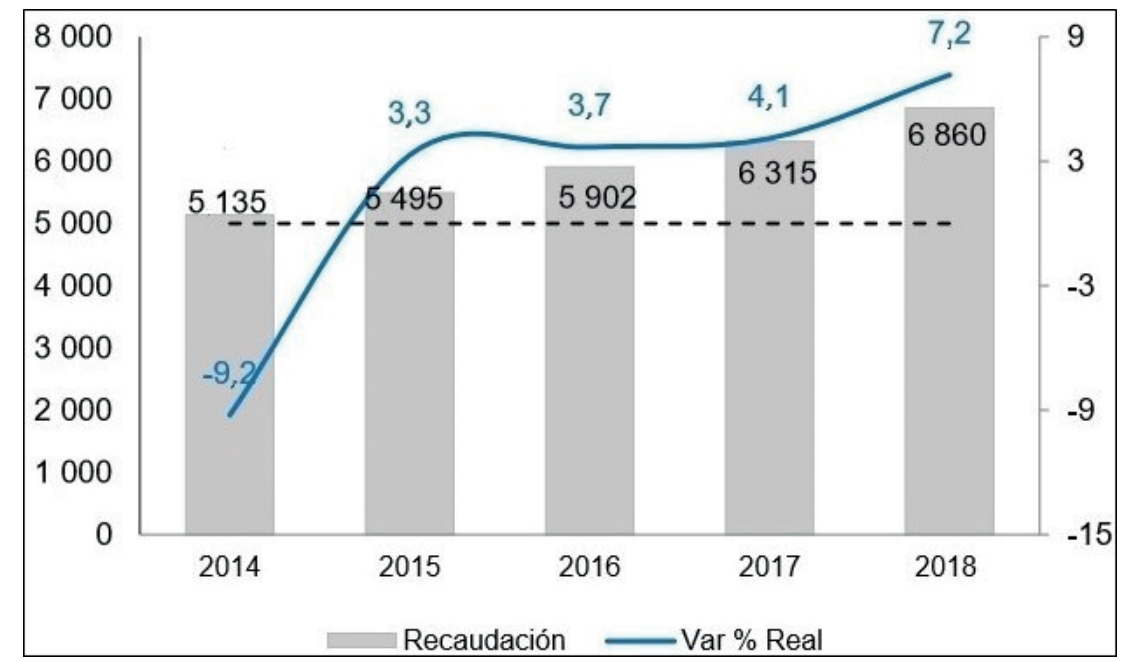

Figura 3. Impuesto Selectivo al Consumo: 2014 - 2018 (En millones de soles y Var. \% real anual).

Fuente: SUNAT: División de Estadística - Gerencia de Estudios Económicos.

Tabla 2

Precios Promedio al Consumidor en Soles años 2015-2019 en Lima Metropolitana con Variación porcentual.

\begin{tabular}{ccccccc}
\hline AÑo & $\begin{array}{c}\text { Gasesosa (SI } \\
\text { por Botella } \\
\text { Vidrio Mediana) }\end{array}$ & $\begin{array}{c}\text { Variación } \\
\%\end{array}$ & $\begin{array}{c}\text { Cerveza Blanca } \\
\text { (S/ por Litro) }\end{array}$ & $\begin{array}{c}\text { Variacón } \\
\%\end{array}$ & $\begin{array}{c}\text { Gasolina de 84 } \\
\text { Octanos (S/por } \\
\text { Galón) }\end{array}$ & $\begin{array}{c}\text { Variación } \\
\%\end{array}$ \\
\hline 2014 & 1,04 & - & 5,80 & - & 13,36 & - \\
2015 & 1,06 & 1,92 & 6,00 & 3,45 & 10,66 & $-20,21$ \\
2016 & 1,10 & 3,77 & 6,14 & 2,33 & 9,34 & $-12,38$ \\
2017 & 1,11 & 0,91 & 6,48 & 5,54 & 10,44 & 11,78 \\
2018 & 1,15 & 3,60 & 6,93 & 6,94 & 11,48 & 9,96 \\
2019 (ENE-MAY) & 1,19 & 3,48 & 7,63 & 10,10 & 11,50 & 0,17 \\
\hline
\end{tabular}

Fuente: Elaboración propia, sobre datos presentados por el Instituto Nacional de Estadística e Informática. 
verado por Villanueva (2014) al indicar que la demanda de los productos gravados se ve mínimamente afectada ante el aumento de sus precios y a su vez, se corrobora lo manifestado por Arias y Plazas (2002) al señalar como uno de los fundamentos de la imposición selectiva al consumo, el representar una fuente importante de ingresos para el Estado. A diferencia del IGV, la legislación vigente respecto a la estructura técnica del ISC, no contempla la traslación económica del impuesto, es decir, el ISC tiene como característica el ser un impuesto monofásico (se grava una sola fase de la cadena productiva). En este sentido, si bien los incrementos de los precios mostrados en la Tabla 2, pueden no deberse exclusivamente al alza en las tasas del impuesto, ello no deja de ser un indicador de que los contribuyentes tienen la libertad para trasladar el impuesto (si así lo deseasen) al consumidor, sin que la cantidad demandada de los productos se vea afectada, como se señaló anteriormente. En este sentido, si los bienes afectos al ISC se caracterizan económicamente por poseer demanda inelástica, carece de lógica pretender disminuir su consumo mediante el aumento de los precios de estos bienes secundado por el incremento de sus tasas impositivas. Lo único que se logra con ello en los hechos es, por un lado, aumentar la recaudación del tributo $y$, por el otro, que el contribuyente de jure traslade el gravamen al consumidor final a través del precio de venta, convirtiendo a este último en el contribuyente de facto del impuesto en mención. Como reflexión final, en la práctica la imposición selectiva se ve distorsionada en cuanto a los fundamentos teóricos que la sustentan, es decir, el impuesto no está cumpliendo su finalidad extrafiscal (desmotivar el consumo de los bienes afectos con el gravamen) sino en cambio, casi por inercia y apoyado en las características económicas de los bienes que son sujetos con el gravamen, el fin fiscal está superando a la extrafiscalidad tradicional que debe perseguir la imposición selectiva. Por lo tanto, los beneficios teóricos que deberían generar en la sociedad este tipo de tributos no se están cumpliendo en nuestro país.

\section{REFERENCIAS BIBLIOGRÁFICAS}

Alva, M. (2017). Aplicación práctica del IGV e ISC. Lima: Pacífico Editores.

Arias, L., \& Plazas, M. (2002). La armonización tributaria de los impuestos indirectos en los países de la Comunidad Andina. Recuperado de intranet.comunidadandina.org/documentos/DInformativos/SGdi428.doc

Congreso de la República de Perú. (2007). Ley Nº 29158 Ley Órganica del Poder Ejecutivo. Lima: Congreso de la República de Perú. Recuperado de http://www.leyes. congreso.gob.pe/Documentos/Leyes/29158.pdf
Hernández, R., \& Mendoza, C. (2018). Metodología de la investigación: Las rutas cuantitativa, cualitativa y mixta. México D.F: McGraw-Hill Interamericana Editores.

Ministerio de Economía y Finanzas. (1999). Decreto Supremo $N^{\circ}$ 055-99-EF Texto Único Ordenado de la Ley del Impuesto General a las Ventas e Impuesto Selectivo al Consumo. Lima: Ministerio de Economía y Finanzas. Recuperado de https:/www.mef.gob.pe/es/ por-instrumento/decreto-supremo/826-d-s-n-05599-ef/file

Ministerio de Economía y Finanzas. (2018). Decreto Supremo $N^{\circ}$ 091-2018-EF Modifican el Literal A del Nuevo Apéndice IV del Texto Único Ordenado de la Ley del Impuesto General a las Ventas e Impuesto Selectivo al Consumo. Lima: Ministerio de Economía y Finanzas. Recuperado de https://cdn.www.gob.pe/uploads/document/file/40385/DS091_2018EF.pdf

Ministerio de Economía y Finanzas. (2018). Decreto Supremo $N^{\circ}$ 092-2018-EF Modifican el Literal B del Nuevo Apéndice IV del Texto Único Ordenado de la Ley del Impuesto General a las Ventas e Impuesto Selectivo al Consumo. Lima: Ministerio de Economía y Finanzas. Recuperado de https://cdn.www.gob.pe/uploads/document/file/40386/DS092_2018EF.pdf

Ministerio de Economía y Finanzas. (2018). Decreto Supremo $N^{\circ}$ 093-2018-EF Modifican la Tabla el Literal D del Nuevo Apéndice IV del Texto Único Ordenado de la Ley del Impuesto General a las Ventas e Impuesto Selectivo al Consumo. Lima: Ministerio de Economía y Finanzas. Recuperado de https://cdn.www.gob.pe/ uploads/document/file/40387/DS093_2018EF.pdf

Ministerio de Economía y Finanzas. (2018). Decreto Supremo $N^{\circ}$ 094-2018-EF Modifican el Nuevo Apéndice III del Texto Único Ordenado de la Ley del Impuesto General a las Ventas e Impuesto Selectivo al Consumo. Lima: Ministerio de Economía y Finanzas. Recuperdo de https://cdn.www.gob.pe/uploads/document/ file/40388/DS094_2018EF.pdf

Ministerio de Economía y Finanzas. (2018). Decreto Supremo $N^{\circ}$ 095-2018-EF Modifican el Literal A del Nuevo Apéndice IV del Texto Único Ordenado de la Ley del Impuesto General a las Ventas e Impuesto Selectivo al Consumo. Lima: Ministerio de Economía y Finanzas. Recuperado de https://cdn.www.gob.pe/uploads/document/file/40389/DS095_2018EF.pdf

Ruiz, F. (2017). Derecho tributario: Temas básicos. Lima: Fondo Editorial Pontifica Universidad Católica del Perú. 
Superintendencia Nacional de Aduanas y de Administración Tributaria. (2018). Memoria Anual 2017: Información para la cuenta General de la República. Recuperado de http://www.sunat.gob.pe/cuentassunat/ planestrategico/memoria/memoria2017.pdf

Superintendencia Nacional de Aduanas y de Administración Tributaria. (2019). Memoria Institucional 2018.
Recuperado de http://www.sunat.gob.pe/cuentassunat/planestrategico/memoria/memoria2018.pdf

Villanueva, W. (2014). Tratado del IGV: Regímenes general y especiales. Lima: Pacífico Editores.

Villegas, H. (2001). Curso de finanzas, derecho financiero y tributario. Buenos Aires: Ediciones Depalma 